\title{
Características de carcaça de cordeiros Ideal e cruzas Border Leicester X Ideal submetidos a três sistemas alimentares
}

\author{
Carcass characteristics of Polwarth and Border Leicester crossbred lambs in three feed systems
}

\author{
Henrique Silla Lopes de Almeida ${ }^{1}$ Cléber Cassol Pires ${ }^{2}$ Diego Barcelos Galvani ${ }^{3}$ \\ Raquel Franco de Lima ${ }^{1}$ Marcel Hastenpflug ${ }^{3}$ Bernardo Garziera Gasperin ${ }^{4}$
}

\section{RESUMO}

O experimento foi realizado com intuito de avaliar o efeito do cruzamento entre as raças Ideal e Border Leicester sobre as características da carcaça de cordeiros submetidos a distintos sistemas alimentares. Foram utilizados 48 cordeiros, machos não castrados, desmamados em média aos 60 dias de idade, sendo: 16 da raça Ideal (I), 16 cruzados 1/2 Border Leicester 1/2 Ideal (BI) e 16 cruzados 3/4 Border Leicester 1/4 Ideal $(B B I)$, aleatoriamente distribuídos em três sistemas de alimentação: pastagem cultivada (PC), pastagem cultivada + suplementação (PCS) e pastagem natural + suplementação (PNS). O abate ocorreu quando os animais atingiram $65 \%$ do peso vivo adulto de seu grupo genético. Houve interação significativa entre genótipos e sistemas de alimentação somente para a variável peso de costela. Foi verificado efeito significativo do cruzamento para as variáveis peso de carcaça quente (PCQ), rendimento de carcaça quente (RCQ), peso de carcaça fria (PCF), rendimento de carcaça fria (RCF), área de olho de lombo (AOL), pesos de paleta (PAL) e peso perna (PER). O cruzamento entre as raças Border Leicester e Ideal resultou em pesos e rendimentos de carcaça superiores e redução da deposição de gordura nas carcaças.

Palavras-chave: cruzamentos, pastagens, ovinos, produção de carne, suplementação.

\section{ABSTRACT}

The experiment was carried out to study the effect of crossing Border Leicester and Polwarth breeds on carcass characteristics of lambs submitted to different feed systems. Forty-eight lambs, non-castrate males, weaned averaging 60 days of age were used: 16 Polwarth (P), 16 1/2 Border Leicester $1 / 2$ Polwarth (BP) and 16 3/4 Border Leicester 1/4 Ideal (BBP).
Animals were randomly distributed in three feed systems: cultivated pasture (CP), cultivated pasture + supplementation (CPS) and natural pasture + supplementation (NPS). The lambs were slaughtered when they reached $65 \%$ of the adult live weight of their genetic groups. Genotype $X$ feed systems interaction was observed only for rib weight. It was verified significant effect of the crossing for the variables hot carcass weight (HCW), hot carcass yield (HCY), cold carcass weight (CCW), cold carcass yield (CCY), loin eye area (LYA), sholder weight $(P W)$ and leg weight $(L W)$. The crossbreeding between the Border Leicester and Polwarth resulted in superior carcass weight and yields and reduction of the deposition of fat.

Key words: crossbreeding, pastures, meat production, sheep, supplementation.

\section{INTRODUÇÃO}

Nas duas últimas décadas, a crise no mercado mundial da lã provocou a desestruturação da cadeia produtiva e forçou o redirecionamento de parte do rebanho ovino gaúcho para a produção de carne. Desde então, inúmeros trabalhos foram desenvolvidos visando a potencializar a produção de carne ovina, sendo esta uma atividade com excelente potencial de desenvolvimento e geração de renda.

A utilização de cruzamentos com raças de aptidão para produção de carne interfere em características quantitativas e qualitativas da carcaça, melhorando suas características de conformação e,

${ }^{1}$ Curso de Pós-graduação em Zootecnia, Universidade Federal de Santa Maria (UFSM), Santa Maria, RS, Brasil. E-mail: henriquelalmeida@yahoo.com; raquelfl@yahoo.com.br.

${ }^{2}$ Departamento de Zootecnia, UFSM, Campus Camobi, 97105-900, Santa Maria, RS, Brasil. E-mail: cpires@ccr.ufsm.br. Autor para correspondência.

${ }^{3}$ Curso de Zootecnia, UFSM, Santa Maria, RS, Brasil.

${ }^{4}$ Curso de Medicina Veterinária, UFSM, Santa Maria, RS, Brasil. 
portanto, de rendimento, bem como acarreta uma melhor distribuição da gordura (SAÑUDO et al., 1997). No entanto, a utilização de cruzamentos desorientados durante anos reduziu substancialmente a qualidade da lã produzida, o que atualmente passa a ser indesejável, tendo em vista os recentes sinais de recuperação demonstrados pelo mercado laneiro. BIANCHI (1998), citando o exemplo australiano, salientou ser possível compatibilizar a produção de carne e lã, obtendo produtos de qualidade, apenas através do correto planejamento dos cruzamentos.

A utilização de ventres de aptidão laneira que apresentam velos fora dos padrões da raça em cruzamentos com raças de carne deve ser considerada na produção de cordeiros, pois possibilita reduzir o tempo de acabamento e melhora significativamente a qualidade de carcaça destes animais (CUNHA et al., 2000). De acordo com KLEEMAN \& DOLLING (1978), os cruzamentos utilizando carneiros da raça Border Leicester e ovelhas de raças especializadas na produção de lã produzem cordeiros mais pesados, com melhor conversão alimentar e mais prolíficos, havendo, ainda, a produção de velos com boa aceitação nos mercados consumidores. Segundo esses autores, este tipo de cruzamento é amplamente utilizado na Austrália, onde constitui a base da indústria de carne ovina.

Por outro lado, é de fundamental importância que os sistemas de produção utilizados sejam compatíveis com a realidade da pecuária gaúcha. $\mathrm{O}$ objetivo deste trabalho foi avaliar o efeito do cruzamento entre as raças Ideal e Border Leicester sobre as características da carcaça dos cordeiros, sendo estes submetidos a três sistemas alimentares.

\section{MATERIAL E MÉTODOS}

O experimento foi conduzido em duas etapas, no período compreendido entre setembro e dezembro de 2002. A primeira, correspondente à fase de terminação dos animais, foi realizada na fazenda Santa Vitória, localizada no município de São Borja, o qual está situado na região fisiográfica denominada Missões, no Estado do Rio Grande do Sul, a $28^{\circ} 20^{\prime} 00^{\prime}$ de latitude Sul e 55¹8'25” de longitude oeste. O clima da região, de acordo com a classificação de Köppen, é do tipo Cfa, subtropical úmido, com temperatura média anual de $19,6^{\circ} \mathrm{C}$ e precipitação pluviométrica anual de $1.598 \mathrm{~mm}$, em média (MORENO, 1961). A segunda etapa do experimento, correspondente ao abate e posterior avaliação das carcaças e seus componentes, foi realizada no Laboratório de Ovinocultura do
Departamento de Zootecnia da Universidade Federal de Santa Maria, RS, Brasil.

Foram utilizados 48 cordeiros, machos nãocastrados, desmamados em média aos 60 dias de idade, sendo: 16 da raça Ideal (I), 16 cruzados 1/2 Border Leicester $1 / 2$ Ideal (BI) e 16 cruzados 3/4 Border Leicester 1/4 Ideal (BBI), distribuídos aleatoriamente, de acordo com o grupo genético, nos seguintes sistemas alimentares: pastagem cultivada (PC), pastagem cultivada + suplementação (PCS) e pastagem natural + suplementação (PNS). No início do período experimental, os animais apresentaram pesos iniciais de 12,1, 12,5 e 17,1kg, respectivamente para I, BI e BBI.

A pastagem cultivada foi composta por azevém (Lolium multiflorum Lam. cv. “Comum”), trevo vermelho (Trifolium pratense L. cv. "Redland II”) e cornichão “El rincón” (Lotus subbiflorus cv. "El Rincón”). A pastagem natural estava composta predominantemente pelos gêneros Paspalum, Axonopus, Sporobolus, Aristida, Eragostris, Andropogon, Coelorhachis, Stipa, Desmodium, Trifolium e espécies das famílias Compositeae e Ciperaceae. As massas e as ofertas de forragem observadas durante o período experimental foram de $840 \mathrm{~kg} \mathrm{ha}^{-1} \mathrm{MS}$ e $9,38 \%$ (PCS), $1074 \mathrm{~kg} \mathrm{ha}^{-1}$ MS e 9,37\% (PC) e $763 \mathrm{~kg} \mathrm{ha}^{-1} \mathrm{MS}$ e $9,91 \%$ (PNS), respectivamente. Os animais dos sistemas alimentares suplementados receberam concentrado comercial com aproximadamente $18 \%$ de proteína bruta, oferecido ao nível de 1,5\% do peso vivo/dia, dividido em partes iguais fornecidas às $8 \mathrm{~h}$ e $18 \mathrm{~h}$.

O abate ocorreu ao serem atingidos $65 \%$ do peso vivo adulto de cada grupo genético, ou seja, a uma mesma maturidade fisiológica, o que correspondeu a 28,0kg para I, 30,5kg para BI e 31,0kg para BBI. Previamente, os animais foram submetidos a um jejum de sólidos e líquidos por $12 \mathrm{~h}$, com posterior pesagem para determinação do peso ao abate (PA) e sangria por meio da secção das veias carótidas e jugulares.

As carcaças foram pesadas para determinação do peso de carcaça quente (PCQ) e rendimento de carcaça quente (RCQ), sendo, então, acondicionadas em câmara fria a $4^{\circ} \mathrm{C}$ por 24 horas, quando foram novamente pesadas para determinação do peso de carcaça fria (PCF) e do rendimento de carcaça fria (RCF). Posteriormente, as carcaças foram cortadas transversalmente entre a $12^{\mathrm{a}}$ e a $13^{\mathrm{a}}$ costelas para exposição do músculo Longissimus dorsi e avaliação da área de olho de lombo (AOL), da espessura de gordura (EG) e do marmoreio (MARM) (1- inexistente a 5 - excessivo). Foram realizadas, 
ainda, avaliações do estado de engorduramento (EE) (1- excessivamente magra a 5- excessivamente gorda) e de conformação das carcaças (CONF) (1- muito pobre a 5- excelente).

Em seguida, as carcaças foram seccionadas longitudinalmente e, após pesagem das meias carcaças esquerdas (PMC), foram determinadas as medidas de comprimento de carcaça (CC), de comprimento de perna (CP), de largura de perna (LP), de profundidade de perna (PP) e de profundidade de peito (PPT). Posteriormente, foram retirados e pesados o pescoço (PES), a cauda (CAU), a paleta (PAL), a costela (COST) e a perna (PER), sendo suas proporções determinadas em relação à meia carcaça esquerda. As avaliações citadas foram realizadas conforme metodologia sugerida por OSÓRIO et al. (1998).

O delineamento experimental utilizado foi o inteiramente casualizado, num arranjo fatorial 3x3 (3 genótipos x 3 sistemas de alimentação), no qual cada animal constituiu uma observação, havendo duas repetições de piquete para cada sistema alimentar. Os dados foram submetidos à análise de variância e suas médias comparadas pelo teste de Tukey ao nível de significância de 5\%, com auxílio do pacote estatístico SAS, versão 6 (SAS, 1997).

\section{RESULTADOS E DISCUSSÃO}

Os valores médios para peso ao abate (PA), peso de carcaça quente (PCQ), rendimento de carcaça quente (RCQ), peso de carcaça fria (PCF), rendimento de carcaça fria (RCF), conformação (CONF), estado de engorduramento (EE), marmoreio (MARM), espessura de gordura (EG), comprimento de carcaça (CC), comprimento de perna (CP), profundidade da perna (PP), largura de perna (LP), profundidade de peito (PPT) e área de olho de lombo (AOL) estão apresentados na tabela 1.

Não foi observada interação significativa $(\mathrm{P}>0,05)$ entre genótipos e sistemas alimentares. Os animais cruzados apresentaram valores de PA superiores $(\mathrm{P}<0,05)$, quando comparados aos animais puros, diferenças estas que já eram esperadas em função de os animais terem sido abatidos em uma mesma maturidade fisiológica, o que significou diferentes pesos de abate para cada grupo genético. Também as médias de PCQ, RCQ, PCF e RCF foram superiores $(\mathrm{P}<0,05)$ para os animais cruzados em relação aos animais da raça Ideal; no entanto, não diferiram entre si. De acordo com OSÓRIO et al. (1996), genótipos

Tabela 1 - Médias e coeficientes de variação para peso ao abate (PA), peso de carcaça quente (PCQ), rendimento de carcaça quente (RCQ), peso de carcaça fria (PCF), rendimento de carcaça fria (RCF), espessura de gordura (EG), comprimento de carcaça (CC), comprimento de perna (CP), profundidade da perna (PP), largura de perna (LP), profundidade de peito (PPT), área de olho de lombo (AOL), conformação (CONF), estado de engorduramento (EE) e marmoreio (MARM).

\begin{tabular}{|c|c|c|c|c|c|c|c|}
\hline \multirow{2}{*}{ Parâmetros } & \multicolumn{3}{|c|}{ Genótipo } & \multicolumn{3}{|c|}{ Alimentação } & \multirow{2}{*}{ CV (\%) } \\
\hline & BBI & BI & I & PCS & PC & PNS & \\
\hline PA (kg) & $28,468^{\mathrm{A}}$ & $27,834^{\mathrm{A}}$ & $25,403^{B}$ & 27,610 & 26,584 & 27,436 & 6,53 \\
\hline PCQ (kg) & $12,736^{\mathrm{A}}$ & $12,295^{\mathrm{A}}$ & $10,658^{\mathrm{B}}$ & 12,258 & 11,565 & 11,759 & 8,63 \\
\hline RCQ (\%) & $44,87^{\mathrm{A}}$ & $44,13^{\mathrm{A}}$ & $41,95^{\mathrm{B}}$ & 44,46 & 43,44 & 42,90 & 4,74 \\
\hline PCF (kg) & $11,608^{\mathrm{A}}$ & $11,768^{\mathrm{A}}$ & $9,848^{\mathrm{B}}$ & $11,672^{\mathrm{a}}$ & $10,514^{b}$ & $10,918^{\mathrm{ab}}$ & 8,05 \\
\hline RCF (\%) & $40,86^{\mathrm{A}}$ & $42,40^{\mathrm{A}}$ & $38,78^{\mathrm{B}}$ & $42,13^{\mathrm{a}}$ & $39,78^{\mathrm{ab}}$ & $39,70^{\mathrm{b}}$ & 5,34 \\
\hline $\mathrm{EG}(\mathrm{mm})$ & $1,62^{\mathrm{C}}$ & $1,71^{\mathrm{B}}$ & $1,81^{\mathrm{A}}$ & 1,69 & 1,96 & 1,50 & 16,48 \\
\hline $\mathrm{CC}(\mathrm{cm})$ & $55,43^{A}$ & $55,16^{\mathrm{A}}$ & $53,20^{\mathrm{B}}$ & 54,61 & 54,34 & 54,84 & 3,87 \\
\hline $\mathrm{CP}(\mathrm{cm})$ & $36,58^{\mathrm{A}}$ & $35,25^{\mathrm{B}}$ & $35,72^{\mathrm{AB}}$ & 35,95 & 35,94 & 35,65 & 2,76 \\
\hline $\mathrm{PP}(\mathrm{cm})$ & $13,43^{\mathrm{A}}$ & $13,20^{\mathrm{AB}}$ & $12,06^{\mathrm{B}}$ & 13,38 & 12,44 & 12,76 & 10,33 \\
\hline $\mathrm{LP}(\mathrm{cm})$ & $8,77^{\mathrm{A}}$ & $9,06^{\mathrm{A}}$ & $7,88^{\mathrm{B}}$ & 8,81 & 8,36 & 8,48 & 8,44 \\
\hline PPT (cm) & $24,68^{\mathrm{A}}$ & $24,25^{\mathrm{AB}}$ & $23,96^{\mathrm{B}}$ & 24,65 & 24,22 & 23,95 & 2,63 \\
\hline $\mathrm{AOL}(\mathrm{cm})$ & $11,23^{\mathrm{A}}$ & $11,39^{\mathrm{A}}$ & $9,13^{\mathrm{B}}$ & 11,41 & 10,05 & 10,11 & 14,80 \\
\hline CONF (1-5) & $3,30^{\mathrm{A}}$ & $3,00^{\mathrm{AB}}$ & $2,80^{\mathrm{B}}$ & 3,10 & 3,00 & 3,00 & 11,94 \\
\hline EE (1-5) & 3,30 & 3,40 & 3,00 & 3,40 & 3,20 & 3,00 & 15,08 \\
\hline MARM (1-5) & $2,90^{\mathrm{AB}}$ & $2,70^{\mathrm{B}}$ & $3,00^{\mathrm{A}}$ & 2,70 & 3,00 & 3,00 & 10,93 \\
\hline
\end{tabular}

Valores seguidos com letras diferentes na mesma linha diferem significativamente pelo teste de Tukey $(\mathrm{P}<0,05)$.

$\mathrm{BBI}=3 / 4$ Border Leicester $1 / 4$ Ideal

$\mathrm{BI}=1 / 2$ Border Leicester $1 / 2$ Ideal

$\mathrm{I}=$ Ideal

PCS = pastagem cultivada + suplementação

$\mathrm{PC}=$ pastagem cultivada

PNS = pastagem natural + suplementação

Ciência Rural, v.36, n.5, set-out, 2006. 
de maior aptidão para produção de carne tendem a possuir melhores rendimentos de carcaça, quando comparados a outras raças menos especializadas para este propósito, o que justifica o resultado encontrado neste estudo.

Da mesma forma, as médias de comprimento de carcaça, de largura de perna e de área de olho de lombo foram significativamente superiores $(\mathrm{P}<0,05)$ para os animais cruzados, corroborando os resultados encontrados por PILAR (2002), em trabalho realizado com cordeiros Merino e suas cruzas com Ile de France.

Os valores encontrados para espessura de gordura de cobertura apresentaram diferenças significativas $(\mathrm{P}<0,05)$ entre os genótipos estudados, sendo que maiores espessuras coincidem com maiores concentrações do genótipo Ideal. As diferenças encontradas para esta variável concordam com SNOWDER et al. (1994), que afirmam que grupos raciais com maturidade precoce apresentam maior cobertura de gordura quando comparados com genótipos de maturidade mais tardia. LEE (1996) observou que cordeiros Merino Australiano apresentavam maior cobertura de gordura quando comparados às suas cruzas com Border Leicester.

Os cordeiros BBI apresentaram medidas superiores $(\mathrm{P}<0,05)$ para profundidade de perna e profundidade de peito em comparação com os cordeiros I, sendo o genótipo BI semelhante aos demais $(\mathrm{P}>0,05)$. Trabalhando com cordeiros de ambos os sexos, filhos de carneiros Ile de France ou Suffolk com ovelhas Ideal, CUNHA et al. (2000) observaram que os cordeiros machos não apresentaram diferenças significativas para profundidade de peito e perímetro de perna entre os genótipos estudados, porém resultados significativos em favor dos cruzados foram observados entre as cordeiras.

As carcaças dos cordeiros BBI apresentaram melhor conformação em relação àquelas dos cordeiros I $(\mathrm{P}<0,05)$; contudo, as carcaças dos cordeiros BI não diferiram das demais $(\mathrm{P}>0,05)$. Este resultado deve-se à maior aptidão carniceira da raça Border Leicester em comparação com a raça Ideal. Desta forma, na medida em que ocorre um aumento da proporção do genótipo Border Leicester, há uma melhoria na conformação da carcaça destes animais, o que corrobora o trabalho de OSÓRIO et al. (2002a), os quais afirmam que, a conformação está associada ao peso do corpo e da carcaça. O marmoreio mais consistente foi verificado nos cordeiros Ideal $(3,0)$, diferindo significativamente $(\mathrm{P}<0,05)$ dos cordeiros $\mathrm{BI}$, que apresentaram o menor escore para esta característica (2,7). Os cordeiros BBI, que apresentaram escore intermediário $(2,9)$, não diferiram significativamente dos demais $(\mathrm{P}>0,05)$.
As médias de PA, PCQ e RCQ não foram influenciadas significativamente pelos sistemas alimentares $(\mathrm{P}>0,05)$. No entanto, os animais terminados no sistema de pastagem cultivada + suplementação (PCS) apresentaram pesos de carcaça fria significativamente superiores $(\mathrm{P}<0,05)$, quando comparados aos cordeiros terminados exclusivamente em pastagem cultivada (PC). Os animais submetidos a PNS não diferiram $(\mathrm{P}>0,05)$ dos demais para esta variável. De forma semelhante, os cordeiros terminados em PCS apresentaram melhor RCF (42,13\%), diferindo dos animais submetidos ao tratamento PNS (39,70\%) $(\mathrm{P}<0,05)$. Os animais terminados em PC apresentaram média de RCF (39,78\%) semelhante às médias obtidas nos demais sistemas alimentares $(\mathrm{P}>0,05)$. Resultados semelhantes foram obtidos por PRIOLO et al. (2002) que, comparando sistemas de alimentação utilizando cordeiros Ile de France, observaram que os cordeiros que recebiam concentrado obtiveram melhores pesos e rendimentos de carcaça fria (15,8 kg e 55,9\%), quando comparados àqueles terminados exclusivamente em pastagem $(14,7 \mathrm{~kg}$ e 53,0\%). Da mesma forma, trabalhando com cordeiros machos não castrados da raça Talaverana, DÍAZ et al. (2002) concluíram que o uso de concentrado na alimentação destes animais propiciou melhores PCF e RCF. OSÓRIO et al. (2002a) afirmaram que animais terminados exclusivamente em campo natural podem apresentar pior rendimento de carcaça fria em função de um menor aporte energético, com conseqüente menor estado de engorduramento das carcaças. Contudo, no presente estudo, não foram encontradas diferenças significativas $(\mathrm{P}>0,05)$ quanto à espessura de gordura e ao estado de engorduramento das carcaças entre os sistemas alimentares avaliados, discordando de SANTOS-SILVA et al. (2002), os quais observaram que as carcaças com menor engorduramento eram oriundas de animais exclusivamente em pastejo, quando comparadas àquelas de animais recebendo suplementação. Os resultados obtidos neste trabalho podem estar relacionados ao reduzido tempo de permanência dos animais em pastejo, o qual foi de 64, 66 e 75 dias para PCS, PC e PNS.

Os valores médios encontrados para as características morfológicas da carcaça não foram influenciados $(\mathrm{P}>0,05)$ pelos sistemas de alimentação, estando próximos aos encontrados por OSÓRIO et al. (2002b), os quais trabalharam com cordeiros cruza Border Leicester X Ideal. Segundo GARCIA et al. (2000), variações encontradas entre cordeiros contemporâneos são devidas, prioritariamente, a diferenças de genótipo.

Na tabela 2, podem ser observados os valores médios para os pesos da meia carcaça (PMC), 
Tabela 2 - Médias e coeficientes de variação para peso da meia carcaça esquerda fria (PCEF), peso de pescoço (PES), peso de cauda (CAU), peso de paleta (PAL) e peso de perna (PER).

\begin{tabular}{|c|c|c|c|c|c|c|c|}
\hline \multirow{2}{*}{ Parâmetros (kg) } & \multicolumn{3}{|c|}{ Genótipo } & \multicolumn{3}{|c|}{ Alimentação } & \multirow{2}{*}{ CV (\%) } \\
\hline & BBI & $\mathrm{BI}$ & I & PCS & PC & PNS & \\
\hline PMC & $5,849^{A}$ & $5,946^{\mathrm{A}}$ & $4,989^{\mathrm{B}}$ & 5,880 & 5,375 & 5,491 & 8,34 \\
\hline PES & 0,465 & 0,423 & 0,421 & 0,445 & 0,422 & 0,431 & 14,84 \\
\hline CAU & $0,060^{\mathrm{A}}$ & $0,058^{\mathrm{AB}}$ & $0,046^{\mathrm{B}}$ & 0,055 & 0,054 & 0,054 & 11,60 \\
\hline PAL & $1,161^{\mathrm{A}}$ & $1,184^{\mathrm{A}}$ & $0,995^{\mathrm{B}}$ & 1,159 & 1,075 & 1,097 & 8,59 \\
\hline PER & $1,987^{\mathrm{A}}$ & $2,005^{\mathrm{A}}$ & $1,709^{\mathrm{B}}$ & 1,993 & 1,830 & 1,859 & 7,16 \\
\hline
\end{tabular}

Valores seguidos com letras diferentes na mesma linha diferem significativamente pelo teste de Tukey $(\mathrm{P}<0,05)$.

$\mathrm{BBI}=3 / 4$ Border Leicester $1 / 4$ Ideal

$\mathrm{BI}=1 / 2$ Border Leicester $1 / 2$ Ideal

$\mathrm{I}=$ Ideal

PCS = pastagem cultivada + suplementação

$\mathrm{PC}=$ pastagem cultivada

PNS = pastagem natural + suplementação

de pescoço (PES), da cauda (CAU), da paleta (PAL) e da perna (PER), nos distintos genótipos e sistemas alimentares testados. Não houve interação $(P>0,05)$ entre genótipos e sistemas de alimentação. Os valores verificados em PMC foram significativamente superiores $(\mathrm{P}<0,05)$ para os cordeiros cruzados, em relação aos puros. Comportamento semelhante foi observado para os pesos de paleta e de perna. O peso de cauda foi maior nos cordeiros BBI em relação aos I $(P>0,05)$, sendo os animais BI semelhantes aos demais $(P>0,05)$. Não foram observadas diferenças significativas $(\mathrm{P}>0,05)$ entre os grupos raciais para peso de pescoço. Segundo OSÓRIO et al. (2002a), genótipos mais pesados refletem-se em carcaças mais pesadas e, conseqüentemente, em maiores pesos de seus componentes regionais. Embora apresentado muitas vezes como fonte de variação do peso dos componentes regionais da carcaça, o sistema de alimentação, nas condições deste trabalho, não foi responsável por variações nas médias de PCM, PES, CAU, PAL e PER.

Foi verificada interação significativa $(\mathrm{P}<0,05)$ entre os sistemas de alimentação e os genótipos para o peso de costela (COST), conforme pode ser observado na tabela 3 . Os cordeiros cruzados apresentaram valores significativamente superiores $(P<0,05)$ para peso de costela, não diferindo entre si. Também o sistema alimentar representou importante fonte de variação para este componente. A suplementação proporcionou incremento no peso deste parâmetro para os animais $\mathrm{BI}(\mathrm{P}<0,05)$, possivelmente pela maior deposição de gordura nesta região. No entanto, com maiores concentrações do genótipo Border Leicester, somente a suplementação não foi suficiente para desencadear o mesmo processo. Assim, os animais BBI apresentaram peso de costela estatisticamente superior $(\mathrm{P}<0,05)$ aos demais genótipos somente no sistema PCS.

Os diferentes pesos de abate e, conseqüentemente, de carcaça, observados para os distintos genótipos à mesma maturidade fisiológica tornam necessária a avaliação dos componentes da carcaça em termos relativos, tendo como base a meia carcaça. Segundo OSÓRIO et al. (2002a), a comparação percentual dos componentes da carcaça elucida melhor os resultados.

A tabela 4 apresenta os valores percentuais encontrados para as proporções dos componentes regionais da carcaça em relação à meia carcaça

Tabela 3 - Médias e coeficientes de variação para peso de costela (COST).

\begin{tabular}{lcccl}
\hline \multirow{2}{*}{ Tratamentos } & \multicolumn{3}{c}{ COST $(\mathrm{kg})$} & \multirow{2}{*}{ CV (\%) } \\
\cline { 2 - 4 } & PCS & PC & PNS & \\
\hline BBI & $2,411^{\mathrm{Aa}}$ & $1,866^{\mathrm{Ab}}$ & $1,774^{\mathrm{Ab}}$ & 13,96 \\
BI & $2,283^{\mathrm{Aa}}$ & $1,921^{\mathrm{Ab}}$ & $2,237^{\mathrm{Aa}}$ & 22,85 \\
I & $1,738^{\mathrm{B}}$ & $1,599^{\mathrm{B}}$ & $1,626^{\mathrm{B}}$ & 9,15 \\
CV $(\%)$ & 14,77 & 13,76 & 13,50 & - \\
\hline
\end{tabular}

Letras minúsculas diferentes na linha indicam diferença significativa pelo teste de Tukey $(\mathrm{P}<0,05)$.

Letras maiúsculas diferentes na coluna indicam diferença significativa pelo teste de Tukey $(\mathrm{P}<0,05)$.

$\mathrm{BBI}=3 / 4$ Border Leicester $1 / 4$ Ideal.

$\mathrm{BI}=1 / 2$ Border Leicester $1 / 2$ Ideal.

$\mathrm{I}=$ Ideal.

PCS = pastagem cultivada + suplementação.

$\mathrm{PC}=$ pastagem cultivada.

PNS = pastagem natural + suplementação.

Ciência Rural, v.36, n.5, set-out, 2006. 
Tabela 4 - Médias e coeficientes de variação para a proporção de pescoço (PES), cauda (CAU), costela (COST), paleta (PAL) e perna (PER).

\begin{tabular}{|c|c|c|c|c|c|c|c|}
\hline \multirow{2}{*}{ Parâmetros (\%) } & \multicolumn{3}{|c|}{ Genótipo } & \multicolumn{3}{|c|}{ Alimentação } & \multirow{2}{*}{ CV (\%) } \\
\hline & BBI & BI & I & PCS & PC & PNS & \\
\hline PES & $7,83^{\mathrm{AB}}$ & $7,12^{\mathrm{B}}$ & $8,45^{\mathrm{A}}$ & 7,64 & 7,93 & 7,86 & 12,15 \\
\hline CAU & 1,02 & 0,97 & 0,93 & 0,94 & 1,01 & 0,97 & 19,07 \\
\hline COST & $34,65^{\mathrm{AB}}$ & $35,89^{\mathrm{A}}$ & $33,20^{\mathrm{B}}$ & $36,18^{\mathrm{a}}$ & $33,35^{\mathrm{b}}$ & $33,90^{\mathrm{b}}$ & 6,94 \\
\hline PAL & 19,90 & 19,94 & 19,97 & 19,73 & 20,10 & 20,02 & 3,71 \\
\hline PER & 33,98 & 33,80 & 34,24 & 33,92 & 34,17 & 33,96 & 3,75 \\
\hline
\end{tabular}

Valores seguidos com letras diferentes na mesma linha diferem significativamente pelo teste de Tukey $(\mathrm{P}<0,05)$.

$\mathrm{BBI}=3 / 4$ Border Leicester $1 / 4$ Ideal

$\mathrm{BI}=1 / 2$ Border Leicester $1 / 2$ Ideal.

$\mathrm{I}=$ Ideal.

PCS = pastagem cultivada + suplementação.

PC = pastagem cultivada

PNS = pastagem natural + suplementação.

esquerda fria. Os resultados observados evidenciaram a influência do genótipo nas proporções de pescoço e costela. A maior proporção de PES foi verificada nos cordeiros I, sendo estes superiores a $\mathrm{BI}(\mathrm{P}<0,05)$.

Não houve interação entre genótipos e sistemas de alimentação $(\mathrm{P}>0,05)$. Os animais BBI não apresentaram diferença dos demais para nenhuma das variáveis $(\mathrm{P}>0,05)$. Maiores valores percentuais de costela (COST) foram observados nos animais BI, os quais diferiram significativamente de $\mathrm{I}(\mathrm{P}<0,05)$. O maior comprimento de carcaça e profundidade de peito dos animais cruzados pode explicar estes resultados. As proporções dos demais componentes não diferiram entre os genótipos $(\mathrm{P}>0,05)$. Os resultados encontrados neste trabalho foram semelhantes aos encontrados por SOUZA (1993) para as proporções de PES, PAL e PER em cordeiros Romney Marsh. O sistema de alimentação foi causa de variação do percentual de costela, sendo que os cordeiros manejados em PCS apresentaram médias superiores aos demais $(\mathrm{P}<0,05)$, o que, segundo OSÓRIO et al. (2002a), poderia estar associado a uma maior deposição de gordura nesta região. Contudo, este fato não foi observado neste trabalho.

\section{CONCLUSÕES}

Cordeiros provenientes do cruzamento entre as raças Border Leicester e Ideal apresentam rendimento e comprimento de carcaça, largura de perna e a área de olho de lombo maiores e menor quantidade de gordura na carcaça, quando comparados a cordeiros da raça Ideal.

A suplementação de animais em pastejo, utilizando alimentos concentrados, possibilita a obtenção de animais com carcaças mais pesadas.

\section{AGRADECIMENTOS E APRESENTAÇÃO}

Ao Conselho Nacional de Desenvolvimento Científico e Tecnológico (CNPq), pela bolsa concedida aos pesquisadores Diego Barcelos Galvani e Cléber Cassol Pires.

Parte da Dissertação de Mestrado apresentada pelo primeiro autor à Universidade Federal de Santa Maria (UFSM), Santa Maria, RS, Brasil.

\section{REFERÊNCIAS}

BIANCHI, G. Cruzamientos para carne ovina. Revista de la EEMAC, n.13, p.7-17, 1998.

CUNHA, E.A. et al. Utilização de carneiros de corte para obtenção de cordeiros precoces para abate em plantéis produtores de lã. Revista Brasileira de Zootecnia, v.29, n.1, p.243252, 2000.

DÍAZ, M.T. et al. Use of concentrate or pasture for fattening Talaverana breed lambs and its effect on meat quality. Small Ruminant Research, v.43, n.3, p.257-268, 2002.

GARCIA, I.F.F. et al. Características de carcaça de cordeiros Texel x Bergamácia, Texel x Santa Inês e Santa Inês Puros, terminados em confinamento, com casca de café como parte da dieta. Revista Brasileira de Zootecnia, v.30, n.3, p.253260, 2000 .

KLEEMAN, D.O.; DOLLING, H.S. Relative efficiency of Merino and Border Leicester X Merino ewes. Australian Journal of Agricultural Research, v.29, n.3, p.605-613, 1978.

LEE, G.J. Growth and carcass composition of ram, crypthorchid and wether Border Leicester X Merino lambs: effects of increasing carcass weight. Australian Journal of Experimental Agriculture, v.26, n.2, p.153-157, 1996.

MORENO, J.A. Clima do Rio Grande do Sul. Porto Alegre: Secretaria da Agricultura, 1961. 41p.

OSÓRIO, J.C.S. et al. Produção de carne em ovinos de cinco genótipos: 2. Componentes do peso vivo. Ciência Rural, v.26, n.3, p.471-475, 1996. 
OSÓRIO, J.C.S. et al. Métodos para avaliação da produção de carne ovina: in vivo, na carcaça e na carne. Pelotas: UFPEL, 1998. 98p.

OSÓRIO, J.C.S. et al. Qualidade, morfologia e avaliação de carcaças. Pelotas: UFPEL, 2002a. 196p.

OSÓRIO, J.C.S. et al. Produção de carne em cordeiros cruza Border Leicester com ovelhas Corriedale e Ideal. Revista Brasileira de Zootecnia, v.31, n.3, p.1469-1480, 2002b. (Suplemento).

PILAR, R.C. Desempenho, características de carcaça, composição e alometria dos cortes em cordeiros Merino Australiano e cruza Ile de France x Merino Australiano. 2002. 237f. Tese (Doutorado em Zootecnia) - Universidade Federal de Lavras.

PRIOLO, A. et al. Effect of grass or concentrate feeding systems on lambs carcass and meat quality. Meat Science, v.62, n.2, p.179-185, 2002.
SANTOS-SILVA, J. et al. The effect of genotype, feed system and slaughter weight on the quality of light lambs 1 . Growth, carcass composition and meat quality. Livestock Production Science, v.76, n.2, p.17-25, 2002.

SAÑUDO, C. et al. Breed effect on carcass and meat quality of suckling lambs. Meat Science, v.46, n.4, p.357-365, 1997.

SAS - Statistical Analysis System. SAS User'sGuide: statistics. Versão 6. 4.ed. North Caroline: SAS INSTITUTE, 1997. $846 p$.

SNOWDER, G.D. et al. Carcass characteristics and optimal slaughter weights in four breeds of sheep. Journal of Animal Science, v.72, n.4, p. 932-937, 1994.

SOUZA, O.R.C. Efeito da idade ao abate sobre o rendimento de carcaça e composição física dos principais cortes, em cordeiros da raça Romney Marsh. 1993. $102 \mathrm{f}$. Dissertação (Mestrado em Zootecnia) - Faculdade de Agronomia Eliseu Maciel, Universidade Federal de Pelotas. 